\title{
Subdivision Depth Computation for Extra-Ordinary Catmull-Clark Subdivision Surface Patches
}

\author{
Fuhua Frank Cheng ${ }^{1}$, Gang Chen ${ }^{1}$, and Jun-Hai Yong ${ }^{2}$ \\ ${ }^{1}$ University of Kentucky, Lexington, KY, USA \\ ${ }^{2}$ Tsinghua University, Beijing, China
}

\begin{abstract}
A second order forward differences based subdivision depth computation technique for extra-ordinary Catmull-Clark subdivision surface (CCSS) patches is presented. The new technique improves a previous technique in that the computation of the subdivision depth is based on the patch's curvature distribution, instead of its dimension. Hence, with the new technique, no excessive subdivision is needed for extra-ordinary CCSS patches to meet the precision requirement and, consequently, one can make trimming, finite element mesh generation, boolean operations, and tessellation of CCSSs more efficient.
\end{abstract}

\section{Introduction}

Research work for subdivision surfaces has been done in several important areas, such as surface parametrization [6] 10, 11] 14, surface trimming [7, boolean operations [1], mesh editing [13], and error estimate/control [3] 12. For instance, given an error tolerance, 3] shows how many times the control mesh of a CatmullClark subdivision surface (CCSS) patch should be recursively subdivided so that the distance between the resulting control mesh and the limit surface patch would be less than the error tolerance. This error control technique, called subdivision depth computation, is required in all tessellation based applications of CCSSs. [3]'s subdivision depth computation technique for regular CCSS patches is optimum. However, for an extra-ordinary CCSS patch (a patch with an extraordinary vertex), since the subdivision depth computed by 3 depends on first order forward differences of the control points, its value could be bigger than what it actually should be and, consequently, generates excessive mesh elements for regions that are already flat enough.

In this paper we will present a new subdivision depth computation technique for extra-ordinary CCSS patches. The new technique is based on the second order forward differences of an extra-ordinary patch's control points.. The computed subdivision depth reflects the patch's curvature distribution, not its dimension. Hence, with the new technique, no excessive subdivision is needed for regions that are already flat enough and, consequently, trimming, finite element mesh generation, boolean operations, and tessellation of CCSSs can be made more efficient. 


\section{Problem Formulation and Background}

Given the control mesh of an extra-ordinary CCSS patch and an error tolerance $\epsilon$, the goal here is to compute an integer $d$ so that if the control mesh is iteratively refined (subdivided) $d$ times, then the distance between the resulting mesh and the surface patch is smaller than $\epsilon$. $d$ is called the subdivision depth of the surface patch with respect to $\epsilon$. Before we show the computation technique, we need to define related terms and review related techniques for regular CCSS patches.

\subsection{Catmull-Clark Subdivision Surfaces}

Given a control mesh, a CCSS is generated by iteratively refining (subdividing) the control mesh to form new control meshes 2]. The refining process consists of defining new vertices (face points, edge points and vertex points) and connecting the new vertices to form new edges and faces of a new control mesh. The limit surface of the iteratively refined control meshes is called a subdivision surface because the mesh refining (subdivision) process is a generalization of the uniform bicubic B-spline surface subdivision technique. Therefore, CCSSs include uniform B-spline surfaces and piecewise Bézier surfaces as special cases. Actually CCSSs include non-uniform B-spline surfaces and NURBS surfaces as special cases as well [9]. The control mesh of a CCSS patch and the new control mesh after a refining (subdivision) process are shown in Figures 1(a) and (b), respectively. This is a conceptual drawing, the location shown for a new vertex might not be its exact physical location.
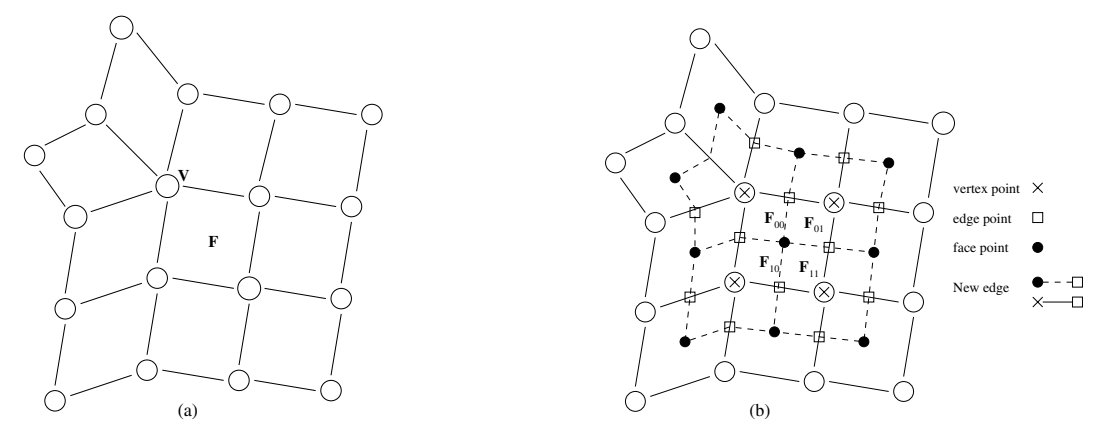

Fig. 1. (a) Control mesh of an extra-ordinary patch; (b) new vertices and edges generated after a Catmull-Clark subdivision

The given control mesh will be referred to as $\mathbf{M}_{0}$ and the limit surface will be referred to as $\overline{\mathbf{S}}$. For each positive integer $k, \mathbf{M}_{k}$ refers to the control mesh obtained after applying the Catmull-Clark subdivision $k$ times to $\mathbf{M}_{0}$.

The power of CCSSs comes from the way mesh vertices are connected. If the number of edges connected to a mesh vertex is called its valence, then the valence of an interior mesh vertex can be anything $\geq 3$, instead of just four. Those mesh vertices whose valences are different from four are called extra-ordinary vertices 
to distinguish them from the standard or regular mesh vertices. Vertex $\mathbf{V}$ in Figure 1(a) is an extra-ordinary vertex of valence five. An interior mesh face is called an extra-ordinary mesh face if it has an extra-ordinary vertex. Otherwise, a standard or regular mesh face. Mesh face $\mathbf{F}$ in Figure $\mathbf{1}(\mathrm{a})$ is an extra-ordinary mesh face. we assume all the mesh faces in $\mathbf{M}_{0}$ are quadrilaterals and each mesh face of $\mathbf{M}_{0}$ has at most one extra-ordinary vertex. Otherwise, simply perform the subdivision step twice on the given control mesh.

For each interior face $\mathbf{F}$ of $\mathbf{M}_{k}, k \geq 0$, there is a corresponding patch $\mathbf{S}$ in the limit surface $\overline{\mathbf{S}}$. $\mathbf{F}$ and $\mathbf{S}$ can be parametrized on the same parameter space $\Omega=[0,1] \times[0,1][10 . \mathbf{F}$ is a bilinear rule surface. $\mathbf{S}$ is a uniform bicubic B-spline surface patch if $\mathbf{F}$ is a regular face. However, if $\mathbf{F}$ is an extra-ordinary face then $\mathbf{S}$, defined by $2 n+8$ control points where $n$ is the valence of $\mathbf{F}$ 's extra-ordinary vertex, can not be parametrized as a uniform B-spline patch. In such a case, $\mathbf{S}$ is called an extra-ordinary patch. Otherwise, a regular patch or standard patch. The control mesh shown in Figure 1(a) is the control mesh of an extra-ordinary patch whose extra-ordinary vertex is of valence five.

\subsection{Distance and Subdivision Depth}

For a given interior mesh face $\mathbf{F}$, let $\mathbf{S}$ be the corresponding patch in the limit surface $\overline{\mathbf{S}}$. The control mesh of $\mathbf{S}$ contains $\mathbf{F}$ as the center face. If we perform a subdivision step on the control mesh, we get four new mesh faces in the place of $\mathbf{F}$. This is the case no matter $\mathbf{F}$ is a regular face or an extra-ordinary face (see Figure 1(b) for the four new faces $\mathbf{F}_{00}, \mathbf{F}_{10}, \mathbf{F}_{01}$ and $\mathbf{F}_{11}$ obtained in the place of the extra-ordinary face $\mathbf{F}$ shown in Figure $\mathbf{1}(\mathrm{a})$ ). Since each of these new faces corresponds to a quarter subpatch of $\mathbf{S}$, we shall call these new faces subfaces of $\mathbf{F}$ even though they are not pyhsically subsets of $\mathbf{F}$. Therefore, each subdivision step generates four new subfaces for the center face $\mathbf{F}$ of the control mesh. Because the correspondence between $\mathbf{F}$ and $\mathbf{S}$ is one-to-one, sometime, instead of saying performing a subdivision step on $\mathbf{S}$, we shall simply say performing a subdivision step on $\mathbf{F}$.

The distance between an interior mesh face $\mathbf{F}$ and the corresponding patch $\mathbf{S}$ is defined as the maximum of $\|\mathbf{F}(u, v)-\mathbf{S}(u, v)\|$ :

$$
D_{\mathbf{F}}=\max _{(u, v) \in \Omega}\|\mathbf{F}(u, v)-\mathbf{S}(u, v)\|
$$

where $\Omega$ is the unit square parameter space of $\mathbf{F}$ and $\mathbf{S}$. $D_{\mathbf{F}}$ is also called the distance between $\mathbf{S}$ and its control mesh. For a given $\epsilon>0$, the subdivision depth of $\mathbf{F}$ with respect to $\epsilon$ is a positive integer $d$ such that if $\mathbf{F}$ is recursively subdivided $d$ times, the distance between each of the resulting subfaces and the corresponding subpatch is smaller than zero.

\subsection{Subdivision Depth Computation for Regular Patches}

A regular patch is a standard uniform bicubic B-spline surface patch. Therefore, the computation process for a regular patch is the same as the computation process for a standard uniform B-spline surface patch. We review the evaluation of the distance between a B-spline patch and its control mesh first. 
Distance Evaluation. Let $\mathbf{S}(u, v)$ be a uniform bicubic B-spline surface patch defined on the unit square $\Omega=[0,1] \times[0,1]$ with control points $\mathbf{V}_{i, j}, 0 \leq$ $i, j \leq 3$, and let $\mathbf{L}(u, v)$ be the bilinear parametrization of the center mesh face $\left\{\mathbf{V}_{1,1}, \mathbf{V}_{2,1}, \mathbf{V}_{2,2}, \mathbf{V}_{1,2}\right\}$ (see Figure 2):

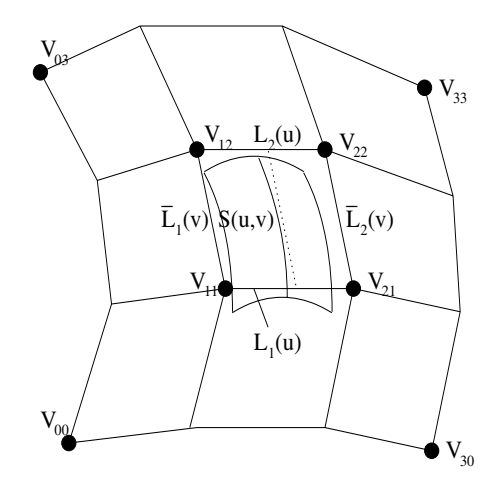

Fig. 2. Definition of $\mathbf{L}(u, v)=(1-v) \mathbf{L}_{1}(u)+v \mathbf{L}_{2}(u)=(1-u) \overline{\mathbf{L}}_{1}(v)+u \overline{\mathbf{L}}_{2}(v)$

$\mathbf{L}(u, v)=(1-v)\left[(1-u) \mathbf{V}_{1,1}+u \mathbf{V}_{2,1}\right]+v\left[(1-u) \mathbf{V}_{1,2}+u \mathbf{V}_{2,2}\right], \quad 0 \leq u, v \leq 1$.

The distance between $\mathbf{S}(u, v)$ and $\mathbf{L}(u, v)$ satisfies the following lemma [3.

Lemma 1. The distance between $\mathbf{L}(u, v)$ and $\mathbf{S}(u, v)$ satisfies the following inequality

$$
\max _{0 \leq u, v \leq 1}\|\mathbf{L}(u, v)-\mathbf{S}(u, v)\| \leq \frac{1}{3} M
$$

where $M$ is the second order norm of $\mathbf{S}(u, v)$ defined as follows

$$
M=\max _{i, j}\left\{\left\|2 \mathbf{V}_{i, j}-\mathbf{V}_{i-1, j}-\mathbf{V}_{i+1, j}\right\|,\left\|2 \mathbf{V}_{i, j}-\mathbf{V}_{i, j-1}-\mathbf{V}_{i, j+1}\right\|\right\}
$$

Recurrence Formula for Second Order Norm. Let $\mathbf{V}_{i, j}, 0 \leq i, j \leq 3$, be the control points of a uniform bicubic B-spline surface patch $\mathbf{S}(u, v)$. We use $\mathbf{V}_{i, j}^{k}$ to represent the new control points of the surface patch after $k$ levels of recursive subdivision. The indexing of the new control points follows the convention that $\mathbf{V}_{0,0}^{k}$ is always the face point of the mesh face $\left\{\mathbf{V}_{0,0}^{k-1}, \mathbf{V}_{1,0}^{k-1}, \mathbf{V}_{1,1}^{k-1}, \mathbf{V}_{0,1}^{k-1}\right\}$. The new control points $\mathbf{V}_{i j}^{k}$ are called the level-k control points of $\mathbf{S}(u, v)$ and the new control mesh will be called the level-k control mesh of $\mathbf{S}(u, v)$.

If we divide the parameter space of the surface patch, $\Omega$, into $4^{k}$ regions as follows:

$$
\Omega_{m n}^{k}=\left[\frac{m}{2^{k}}, \frac{m+1}{2^{k}}\right] \times\left[\frac{n}{2^{k}}, \frac{n+1}{2^{k}}\right], \quad 0 \leq m, n \leq 2^{k}-1
$$

and denote the corresponding subpatches $\mathbf{S}_{m n}^{k}(u, v)$, then each $\mathbf{S}_{m n}^{k}(u, v)$ is a uniform bicubic B-spline surface patch defined by the level- $k$ control point set 
$\left\{\mathbf{V}_{p q}^{k} \mid m \leq p \leq m+3, n \leq q \leq n+3\right\} . \mathbf{S}_{m n}^{k}(u, v)$ is called a level- $k$ subpatch of $\mathbf{S}(u, v)$. Let $\mathbf{L}_{m n}^{k}(u, v)$ be the bilinear parametrization of the center face of $\mathbf{S}_{m n}^{k}$ 's control mesh, $\left\{\mathbf{V}_{p q}^{k} \mid p=m+1, m+2 ; q=n+1, n+2\right\}$. We say the distance between $\mathbf{S}(u, v)$ and the level- $k$ control mesh is smaller than $\epsilon$ if the distance between each level- $k$ subpatch $\mathbf{S}_{m n}^{k}(u, v)$ and the corresponding level- $k$ bilinear plane $\mathbf{L}_{m n}^{k}(u, v), 0 \leq m, n \leq 2^{k}-1$, is smaller than $\epsilon$. A technique to compute a subdivision depth $k$ for a given $\epsilon$ so that the distance between $\mathbf{S}(u, v)$ and the level- $k$ control mesh is smaller than $\epsilon$ is presented in [3]. The following lemma is needed in the derivation of the computation process. If we use $M_{m n}^{k}$ to represent the second order norm of $\mathbf{S}_{m n}^{k}(u, v)$, i.e., the maximum norm of the second order forward differences of the control points of $\mathbf{S}_{m n}^{k}(u, v)$, then the lemma shows the second order norm of $\mathbf{S}_{m n}^{k}(u, v)$ converges at a rate of $1 / 4$ of the level- $(k-1)$ second order norm [3].

Lemma 2. If $M_{m n}^{k}$ is the second order norm of $\mathbf{S}_{m n}^{k}(u, v)$ then we have

$$
M_{m n}^{k} \leq\left(\frac{1}{4}\right)^{k} M
$$

where $M$ is the second order norm of $\mathbf{S}(u, v)$ defined in (2).

Subdivision Depth Computation. With Lemmas 1 and 2, it is easy to see that, for any $0 \leq m, n \leq 2^{k-1}$, we have

$$
\max _{0 \leq u, v \leq 1}\left\|\mathbf{L}_{m n}^{k}(u, v)-\mathbf{S}_{m n}^{k}(u, v)\right\| \leq \frac{1}{3} M_{m n}^{k} \leq \frac{1}{3}\left(\frac{1}{4}\right)^{k} M
$$

where $M_{m n}^{k}$ and $M$ are the second order norms of $\mathbf{S}_{m n}^{k}(u, v)$ and $\mathbf{S}(u, v)$, respectively. Hence, if $k$ is large enough to make the right side of the above inequality smaller than $\epsilon$, we have

$$
\max _{0 \leq u, v \leq 1}\left\|\mathbf{L}_{m n}^{k}(u, v)-\mathbf{S}_{m n}^{k}(u, v)\right\| \leq \epsilon
$$

for every $0 \leq m, n \leq 2^{k-1}$. This leads to the following subdivision depth computation process for a regular CCSS patch $[3]$.

Theorem 3. Let $\mathbf{V}_{i j}, 0 \leq i, j \leq 3$, be the control points of a uniform bicubic B-spline surface patch $\mathbf{S}(u, v)$. For any given $\epsilon>0$, if

$$
k \geq\left\lceil\log _{4}\left(\frac{M}{3 \epsilon}\right)\right\rceil
$$

levels of recursive subdivision are performed on the control points of $\mathbf{S}(u, v)$ then the distance between $\mathbf{S}(u, v)$ and the level- $k$ control mesh is smaller than $\epsilon$ where $M$ is the second order norm of $\mathbf{S}(u, v)$ defined in (2). 


\section{Subdivision Depth Computation for Extra-Ordinary Patches}

In the following, we will define second order forward difference patterns to be used for an extra-ordinary patch and derive a recurrence formula for the corresponding second order norm, like the one used for a regular patch in Section 2.

\subsection{Second Order Norm and Recurrence Formula}

Let $\mathbf{V}_{i}, i=1,2, \ldots, 2 n+8$, be the control points of an extra-ordinary patch $\mathbf{S}(u, v)=\mathbf{S}_{0}^{0}(u, v)$, with $\mathbf{V}_{1}$ being an extra-ordinary vertex of valence $n$. The control points are ordered following J. Stam's fashion [10] (Figure 3(a)). For convenience of subsequent reference, we shall call the control mesh of $\mathbf{S}(u, v)$ $\Pi=\Pi_{0}^{0}$. By performing a subdividion step on $\Pi$, one gets $2 n+17$ new vertices $\mathbf{V}_{i}^{1}, i=1, \ldots, 2 n+17$ (see Figure 3 (b)). These control points form four control point sets $\Pi_{0}^{1}, \Pi_{1}^{1}, \Pi_{2}^{1}$ and $\Pi_{3}^{1}$, representing control meshes of the subpatches $\mathbf{S}_{0}^{1}(u, v), \mathbf{S}_{1}^{1}(u, v), \mathbf{S}_{2}^{1}(u, v)$ and $\mathbf{S}_{3}^{1}(u, v)$, respectively (see Figure 3(b)) where $\Pi_{0}^{1}=\left\{\mathbf{V}_{i}^{1} \mid 1 \leq i \leq 2 n+8\right\}$, and the other three control point sets $\Pi_{1}^{1}, \Pi_{2}^{1}$ and $\Pi_{3}^{1}$ are shown in Figure $4 . \mathbf{S}_{0}^{1}(u, v)$ is an extra-ordinary patch but $\mathbf{S}_{1}^{1}(u, v)$, $\mathbf{S}_{2}^{1}(u, v)$ and $\mathbf{S}_{3}^{1}(u, v)$ are regular patches. Therefore, second order norm similar to (2) can be defined for $\mathbf{S}_{1}^{1}, \mathbf{S}_{2}^{1}$ and $\mathbf{S}_{3}^{1}$.

To define a second order norm for $\mathbf{S}$, one needs to choose appropriate second order forward differences from $\Pi$. For the second order norm to be recursively defined, second order forward differences that are required in the child control meshes should also appear in the parent control mesh. For instance, $2 \mathbf{V}_{1}-$ $\mathbf{V}_{4}-\mathbf{V}_{8}$ and $2 \mathbf{V}_{1}-\mathbf{V}_{2}-\mathbf{V}_{6}$ should be chosen for $\Pi$ because these patterns are required for $\Pi_{1}^{1}$ and $\Pi_{3}^{1}$, respectively. On the other hand, for a recurrence formula to hold effectively, second order forward differences that are not required in the child control meshes should not be used in the parent control mesh either.. For instance, one should not choose $2 \mathbf{V}_{1}-\mathbf{V}_{2}-\mathbf{V}_{8}$ for $\Pi$ because this pattern is not required in any of $\Pi_{1}^{1}, \Pi_{2}^{1}$ or $\Pi_{3}^{1}$. Therefore, for those cases that involves the extra-ordinary point $\mathbf{V}_{1}$ as the center point, one should only consider

$$
2 \mathbf{V}_{1}-\mathbf{V}_{2 i}-\mathbf{V}_{2(i \% n+2)}, \quad 1 \leq i \leq n
$$
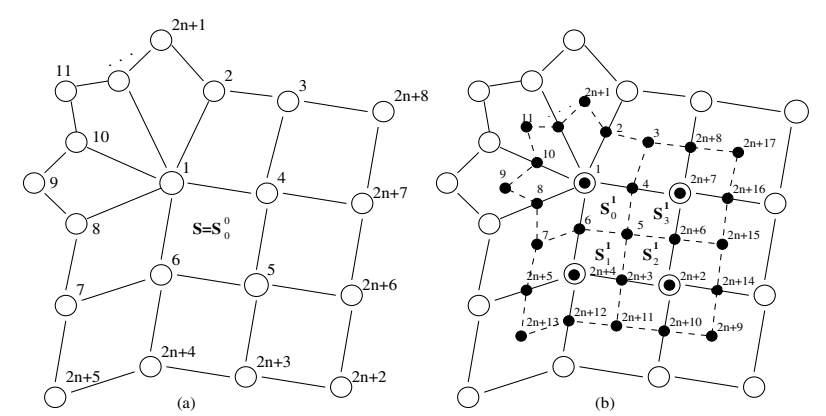

Fig. 3. (a) Ordering of control points of an extra-ordinary patch. (b) Ordering of new control points (solid dots) after a Catmull-Clark subdivision. 


$$
\begin{array}{|c|ccc|c|}
\cline { 2 - 3 } \multicolumn{1}{c|}{\Pi_{1}^{1}} & \mathbf{V}_{2}^{1} & \mathbf{V}_{3}^{1} & \mathbf{V}_{2 n+8}^{1} & \mathbf{V}_{2 n+17}^{1} \\
\hline \mathbf{V}_{8}^{1} & \mathbf{V}_{1}^{1} & \mathbf{V}_{4}^{1} & \mathbf{V}_{2 n+7}^{1} & \mathbf{V}_{2 n+16}^{1} \\
\mathbf{V}_{7}^{1} & \mathbf{V}^{1} & \mathbf{V}_{5}^{1} & \mathbf{V}_{2 n+6}^{1} & \mathbf{V}_{2 n+15}^{1} \\
\mathbf{V}_{2 n+5}^{1} & \mathbf{V}_{2 n+4}^{1} & \mathbf{V}_{2 n+3}^{1} & \mathbf{V}_{2 n+2}^{1} & \mathbf{V}_{2 n+14}^{1} \\
\mathbf{V}_{2 n+13}^{1} & \mathbf{V}_{2 n+12}^{1} & \mathbf{V}_{2 n+11}^{1} & \mathbf{V}_{2 n+1}^{1} & \mathbf{V}_{2 n+9}^{1} \\
\hline
\end{array}
$$

Fig. 4. Control vertices of subpatches $\mathbf{S}_{1}^{1}, \mathbf{S}_{2}^{1}$ and $\mathbf{S}_{3}^{1}$

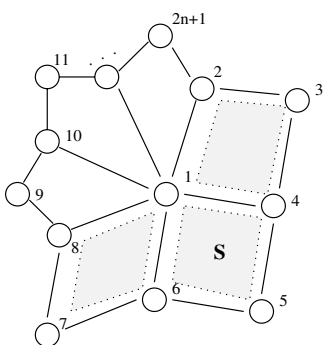

(a)

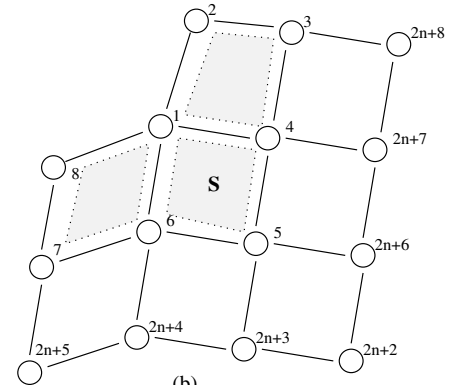

Fig. 5. (a) Vicinity of the extra-ordinary point. (b) The extended remaining part.

To ensure the boundary of the vicinity of the extra-ordinary point is covered (Figure 5(a)), one should consider

$$
2 \mathbf{V}_{2(i \% n+1)}-\mathbf{V}_{2 i+1}-\mathbf{V}_{2(i \% n+1)+1}, \quad 1 \leq i \leq n
$$

One also has to consider second order forward differences that cover the extended remaining part (Figure 5(b)). There are ten of them (actually twelve, but two of them have been used in (6) $)$. So, totally, $2 n+10(n+10$ when $n=3)$ second order forward differences should be considered for $\Pi$ and the second order norm of $\mathbf{S}, M=M_{0}$, is defined as the maximum norm of these $2 n+10$ second order forward differences:

$$
\begin{aligned}
M & =\max \left\{\left\{\left\|2 \mathbf{V}_{1}-\mathbf{V}_{2 i}-\mathbf{V}_{2(i \% n+2)}\right\| \mid 1 \leq i \leq n\right\} \cup\right. \\
& \left\{\left\|2 \mathbf{V}_{2(i \% n+1)}-\mathbf{V}_{2 i+1}-\mathbf{V}_{2(i \% n+1)+1}\right\| \mid 1 \leq i \leq n\right\} \cup \\
& \left\{\left\|2 V_{3}-V_{2}-V_{2 n+8}\right\|,\left\|2 V_{4}-V_{1}-V_{2 n+7}\right\|,\left\|2 V_{5}-V_{6}-V_{2 n+6}\right\|,\right. \\
& \left\|2 V_{5}-V_{4}-V_{2 n+3}\right\|,\left\|2 V_{6}-V_{1}-V_{2 n+4}\right\|,\left\|2 V_{7}-V_{8}-V_{2 n+5}\right\|, \\
& \left\|2 V_{2 n+7}-V_{2 n+6}-V_{2 n+8}\right\|,\left\|2 V_{2 n+6}-V_{2 n+2}-V_{2 n+7}\right\|, \\
& \left.\left.\left\|2 V_{2 n+3}-V_{2 n+2}-V_{2 n+4}\right\|,\left\|2 V_{2 n+4}-V_{2 n+3}-V_{2 n+5}\right\|\right\}\right\}
\end{aligned}
$$


Following this definition, one can define a similar second order norm, $M_{1}$, for the control mesh of $\mathbf{S}_{0}^{1}$. In general, for any $k \geq 0$, we can define second order norm similar to (7) for $\mathbf{S}_{0}^{k}$ and $\mathbf{S}_{0}^{k+1}$. The second order norms of $\mathbf{S}_{0}^{k}$ and $\mathbf{S}_{0}^{k+1}$ are denoted $M_{k}$ and $M_{k+1}$, respectively. We have the following lemma for $M_{k}$ and $M_{k+1}$. The proof is shown in the complete version of the paper 4 .

Lemma 4. For any $k \geq 0$, if $M_{k}$ represents the second order norm of the extraordinary sub-patch $\mathbf{S}_{0}^{k}$ after $k$ Catmull-Clark subdivision steps, then $M_{k}$ satisfies the following inequality

$$
M_{k+1} \leq \begin{cases}\frac{2}{3} M_{k}, & n=3 \\ 0.72 M_{k}, & n=5 \\ \left(\frac{3}{4}+\frac{8 n-46}{4 n^{2}}\right) M_{k}, & n>5\end{cases}
$$

Actually, the lemma works in a more general sense, i.e., if $M_{k}$ stands for the second order norm of the control mesh $\mathbf{M}_{k}$, instead of $\Pi_{0}^{k}$, the lemma still works. The second order norm of $\mathbf{M}_{k}$ is defined as follows: for regions not involving the extra-ordinary point, use standard second order forward differences; for the vicinity of the extra-ordinary point, use second order forward differences defined in (17). The proof is essentially the same.

\subsection{Distance Evaluation}

To compute the distance between the extra-ordinary patch $\mathbf{S}(u, v)$ and the center face of its control mesh, $\mathbf{L}(u, v)$, we need to parameterize the patch $\mathbf{S}(u, v)$ first.

Note that by iteratively performing Catmull-Clark subdivision on $\mathbf{S}(u, v)$, we get a sequence of regular patches $\left\{\mathbf{S}_{b}^{m}\right\}, m \geq 1, b=1,2,3$, and a sequence of extra-ordinary patches $\left\{\mathbf{S}_{0}^{m}\right\}, m \geq 1$. The extra-ordinary patches converge to a limit point which is the value of $\mathbf{S}$ at $(0,0)[5$. This limit point and the regular patches $\left\{\mathbf{S}_{b}^{m}\right\}, m \geq 1, b=1,2,3$, form a partition of $\mathbf{S}$. If we use $\Omega_{b}^{m}$ to represent the parameter space corresponding to $\mathbf{S}_{b}^{m}$ then $\left\{\Omega_{b}^{m}\right\}, m \geq 1$, $b=1,2,3$, form a partition of the unit square $\Omega=[0,1] \times[0,1]$ (see Figure 6 ) with

$$
\begin{aligned}
& \Omega_{1}^{m}=\left[\frac{1}{2^{m}}, \frac{1}{2^{m-1}}\right] \times\left[0, \frac{1}{2^{m}}\right], \quad \Omega_{2}^{m}=\left[\frac{1}{2^{m}}, \frac{1}{2^{m-1}}\right] \times\left[\frac{1}{2^{m}}, \frac{1}{2^{m-1}}\right], \\
& \Omega_{3}^{m}=\left[0, \frac{1}{2^{m}}\right] \times\left[\frac{1}{2^{m}}, \frac{1}{2^{m-1}}\right] .
\end{aligned}
$$

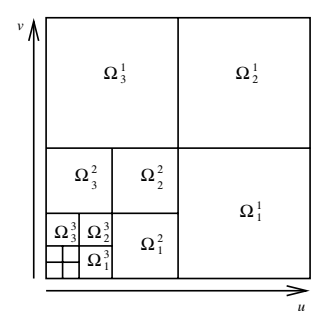

Fig. 6. $\Omega$-partition of the unit square 
The parametrization of $\mathbf{S}(u, v)$ is done as follows. For any $(u, v) \in \Omega$ but $(u, v) \neq$ $(0,0)$, first find the $\Omega_{b}^{m}$ that contains $(u, v)$. $m$ and $b$ can be computed as follows.

$$
\begin{aligned}
& m(u, v)=\min \left\{\left\lceil\log _{\frac{1}{2}} u\right\rceil,\left\lceil\log _{\frac{1}{2}} v\right\rceil\right\} \\
& b(u, v)= \begin{cases}1, & \text { if } 2^{m} u \geq 1 \text { and } 2^{m} v \leq 1 \\
2, & \text { if } 2^{m} u \geq 1 \text { and } 2^{m} v \geq 1 \\
3, & \text { if } 2^{m} u \leq 1 \text { and } 2^{m} v \geq 1\end{cases}
\end{aligned}
$$

Then map this $\Omega_{b}^{m}$ to the unit square with the mapping: $(u, v) \rightarrow\left(u_{m}, v_{m}\right)$ where

$$
t_{m}=\left(2^{m} t\right) \% 1=\left\{\begin{array}{ll}
2^{m} t, & \text { if } 2^{m} t \leq 1 \\
2^{m} t-1, & \text { if } 2^{m} t>1
\end{array} .\right.
$$

The value of $\mathbf{S}(u, v)$ is equal to the value of $\mathbf{S}_{b}^{m}$ at $\left(u_{m}, v_{m}\right)$, i.e., $\mathbf{S}(u, v)=$ $\mathbf{S}_{b}^{m}\left(u_{m}, v_{m}\right)$. Let $\mathbf{L}_{b}^{m}(u, v)$ be the bilinear parametrization of the center face of $\mathbf{S}_{b}^{m}$ 's control mesh. Since $\mathbf{S}_{b}^{m}$ is a regular patch, following Lemma 1, we have

$$
\left\|\mathbf{L}_{b}^{m}(u, v)-\mathbf{S}_{b}^{m}(u, v)\right\| \leq \frac{1}{3} M_{b}^{m}
$$

where $M_{b}^{m}$ is the second order norm of the contol mesh of $\mathbf{S}_{b}^{m}$. But the second order norm of $\mathbf{S}_{b}^{m}$ is smaller than the second order norm of $\mathbf{M}_{m}, M_{m}$. Hence, the above inequality can be written as

$$
\left\|\mathbf{L}_{b}^{m}(u, v)-\mathbf{S}_{b}^{m}(u, v)\right\| \leq \frac{1}{3} M_{m} .
$$

So the maximum distance between the original extra-ordinary mesh $\mathbf{L}(u, v)$ and the patch $\mathbf{S}(u, v)$ can be written as

$$
\begin{aligned}
& \|\mathbf{L}(u, v)-\mathbf{S}(u, v)\|=\left\|\mathbf{L}(u, v)-\mathbf{L}_{b}^{m}\left(u_{m}, v_{m}\right)+\mathbf{L}_{b}^{m}\left(u_{m}, v_{m}\right)-\mathbf{S}(u, v)\right\| \\
& \quad \leq\left\|\mathbf{L}(u, v)-\mathbf{L}_{b}^{m}\left(u_{m}, v_{m}\right)\right\|+\left\|\mathbf{L}_{b}^{m}\left(u_{m}, v_{m}\right)-\mathbf{S}_{b}^{m}\left(u_{m}, v_{m}\right)\right\|
\end{aligned}
$$

where $0 \leq u, v \leq 1$ and $u_{m}$ and $v_{m}$ are defined in (10). Since the second term on the right hand side can be estimated using (11), the only thing we need to work with is $\left\|\mathbf{L}(u, v)-\mathbf{L}_{b}^{m}\left(u_{m}, v_{m}\right)\right\|$.

It is easy to see that if $(u, v) \in \Omega_{b}^{m}$ then $(u, v) \in \Omega_{0}^{k}$ for any $0 \leq k<m$ where $\Omega_{0}^{k}=\left[0, \frac{1}{2^{k}}\right] \times\left[0, \frac{1}{2^{k}}\right] . \Omega_{0}^{k}$ corresponds to the subpatch $\mathbf{S}_{0}^{k}$. This means that $\left(2^{k} u, 2^{k} v\right)$ is within the parameter space of $\mathbf{S}_{0}^{k}$ for $0 \leq k<m$, i.e., $\left(2^{k} u, 2^{k} v\right)=$ $\left(u_{k}, v_{k}\right)$ where $u_{k}$ and $v_{k}$ are defined in (10). Consequently, we can consider $\mathbf{L}_{0}^{k}\left(u_{k}, v_{k}\right)$ for $0 \leq k<m$ where $\mathbf{L}_{0}^{k}$ is the bilinear parametrization of the center face of the control mesh of $\mathbf{S}_{0}^{k}$ (with the understanding that $\mathbf{L}_{0}^{0}=\mathbf{L}$ ). What we want to do here is to write the first term on the right hand side of (12) as

$$
\begin{aligned}
& \mathbf{L}(u, v)-\mathbf{L}_{b}^{m}\left(u_{m}, v_{m}\right)=\mathbf{L}_{0}^{0}(u, v)-\mathbf{L}_{0}^{1}\left(u_{1}, v_{1}\right)+\mathbf{L}_{0}^{1}\left(u_{1}, v_{1}\right)-\mathbf{L}_{0}^{2}\left(u_{2}, v_{2}\right) \\
& \quad+\mathbf{L}_{0}^{2}\left(u_{2}, v_{2}\right)-\mathbf{L}_{0}^{3}\left(u_{3}, v_{3}\right)+\mathbf{L}_{0}^{3}\left(u_{3}, v_{3}\right)-\mathbf{L}_{0}^{4}\left(u_{4}, v_{4}\right) \\
& \quad+\cdots+\mathbf{L}_{0}^{m-1}\left(u_{m-1}, v_{m-1}\right)-\mathbf{L}_{b}^{m}\left(u_{m}, v_{m}\right)
\end{aligned}
$$


and get an estimate for its norm by estimating the norm of each consecutive pair on the right hand side. We have the following two lemmas. The proofs of these lemmas are shown in the complete version of the paper 4 .

Lemma 5. If $(u, v) \in \Omega_{b}^{m}$ where $b$ and $m$ are defined in (9) then for any $0 \leq k<m-1$ we have

$$
\left\|\mathbf{L}_{0}^{k}\left(u_{k}, v_{k}\right)-\mathbf{L}_{0}^{k+1}\left(u_{k+1}, v_{k+1}\right)\right\| \leq \frac{1}{\min \{n, 8\}} M_{k}
$$

where $M_{k}$ is the second order norm of $\mathbf{M}_{k}$ and $\mathbf{L}_{0}^{0}=\mathbf{L}$.

Lemma 6. If $(u, v) \in \Omega_{b}^{m}$ where $b$ and $m$ are defined in (9) then we have

$$
\left\|\mathbf{L}_{0}^{m-1}\left(u_{m-1}, v_{m-1}\right)-\mathbf{L}_{b}^{m}\left(u_{m}, v_{m}\right)\right\| \leq \begin{cases}\frac{1}{4} M_{m-1}, & \text { if } b=2 \\ \frac{1}{8} M_{m-1}, & \text { if } b=1 \text { or } 3\end{cases}
$$

where $M_{m-1}$ is the second order norm of $\mathbf{M}_{m-1}$.

By applying Lemmas 5 and 6 on (13) and then using (11) on (12), we have the following lemma. Proof of this lemma is shown in [4.

Lemma 7. The maximum of $\|\mathbf{L}(u, v)-\mathbf{S}(u, v)\|$ satisfies the following inequality

$$
\|\mathbf{L}(u, v)-\mathbf{S}(u, v)\| \leq \begin{cases}M_{0}, & n=3 \\ \frac{5}{7} M_{0}, & n=5 \\ \frac{4 n}{n^{2}-8 n+46} M_{0}, & 5<n \leq 8 \\ \frac{n^{2}}{4\left(n^{2}-8 n+46\right)} M_{0}, & n>8\end{cases}
$$

where $M=M_{0}$ is the second order norm of the extra-ordinary patch $\mathbf{S}(u, v)$.

Since the coefficient in the third case $\left(4 n /\left(n^{2}-8 n+46\right)\right)$ is smaller than the coefficient in the second case $(5 / 7)$, we can combine these two cases into one case $(5 \leq n \leq 8)$ to make the above expression (14) simpler.

\subsection{Subdivision Depth Computation}

Lemma 7 is important because it not only provides us with a second order norm based simple mechanism to estimate the distance between an extra-ordinary surface patch and its control mesh, it also allows us to estimate the distance between a level- $k$ control mesh and the surface patch for any $k>0$. This is because the distance between a level- $k$ control mesh and the surface patch is dominated by the distance between the level- $k$ extra-ordinary subpatch and the corresponding control mesh which, accoriding to Lemma 7 , is 


$$
\left\|\mathbf{L}_{k}(u, v)-\mathbf{S}(u, v)\right\| \leq \begin{cases}M_{k}, & n=3 \\ 0.72 M_{k}, & 5 \leq n \leq 8 \\ \frac{n^{2}}{4\left(n^{2}-8 n+46\right)} M_{k}, & n>8\end{cases}
$$

where $M_{k}$ is the second order norm of $\mathbf{S}(u, v)$ 's level- $k$ control mesh, $\mathbf{M}_{k}$ (see the remark at the end of Section 3.1 for the definition of $M_{k}$ ). By combining the above result with Lemma 4 , we have the following subdivision depth computation theorem for extra-ordinary surface patches.

Theorem 8. Given an extra-ordinary surface patch $\mathbf{S}(u, v)$ and an error tolerance $\epsilon$, if $k$ levels of subdivisions are iteratively performed on the control mesh of $\mathbf{S}(u, v)$, where

$$
k=\left\lceil\log _{w} \frac{M}{z \epsilon}\right\rceil
$$

with $M$ being the second order norm of $\mathbf{S}(u, v)$ defined in (77),

$$
w=\left\{\begin{array}{ll}
\frac{3}{2}, & n=3 \\
\frac{25}{18}, & n=5 \\
\frac{4 n^{2}}{3 n^{2}+8 n-46}, & n>5
\end{array} \quad \text { and } \quad z= \begin{cases}1, & n=3 \\
\frac{25}{18}, & 5 \leq n \leq 8 \\
\frac{2\left(n^{2}-8 n+46\right)}{n^{2}}, & n>8\end{cases}\right.
$$

then the distance between $\mathbf{S}(u, v)$ and level- $k$ control mesh is smaller than $\epsilon$.

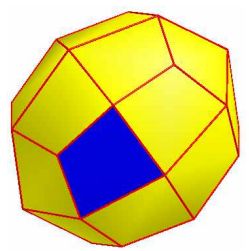

(a)

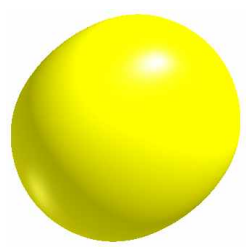

(b)

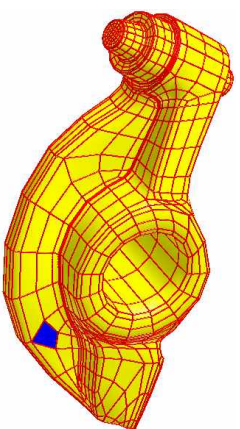

(c)

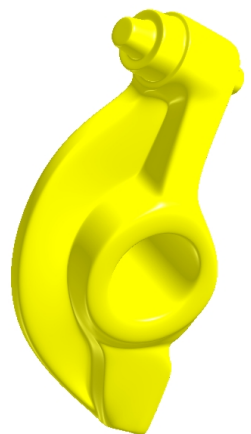

(d)

Fig. 7. Examples: (a) an extra-ordinary CCSS mesh face of valence 3, (b) limit surface of the control mesh shown in (a), (c) an extra-ordinary CCSS mesh face of valence 5, (d) limit surface of the control mesh shown in (c) 


\section{Examples}

Some examples of the presented distance evaluation and subdivision depth computation techniques are given in this section. In Figures 7(a) and 7(c), the distances between the blue mesh faces of the control meshes and the corresponding limit surface patches are 0.16 and 0.81 , respectively. For the blue mesh face shown in Figure 7 (a), the subdivision depths for the error tolerances $0.1,0 . .01$, 0.001 , and 0.0001 are $2,7,13$, and 19, respectively.. For the blue mesh face shown in Figure 7(c), the subdivision depths for the error tolerances 0.1, 0.01, 0.001, and 0.0001 are $7,14,21$, and 28 , respectively. Note that in the previous approach [3], the subdivision depths for these error tolerances are $9,24,40$, and 56, respectively. Hence, the new approach presented in this paper indeed improves the previous, first order norm based approach.

\section{Conclusions}

A new subdivision depth computation technique for extra-ordinary CCSS patches is presented. The new technique computes the subdivision depth based on norms of the second order forward differences, not the first order forward differences, of the patch's control points. Hence, the computed subdivision depth reflects the curvature distribution of the extra-ordinary patch, not its dimension. Our result also points out that as long as the design objective can be achieved, one should try to use extra-ordinary vertices with smaller valence because, according to Theorem 8 , smaller valence gives higher convergence rate and, consequently, smaller subdivision depth for the same precision.

Although the new technique improves the previous approach [3], it is not clear if the new approach is optimum for extra-ordinary CCSS patches. This will be a study direction in the future.

Acknowledgements. Work of the first two authors is supported by NSF under grants DMS-0310645 and DMI-0422126. Work of the third author is supported by NSF of China (60533070), NCET(NCET-04-0088) and FANEDD (200342).

\section{References}

1. Biermann H, Kristjansson D, Zorin D, Approximate Boolean Operations on FreeForm Solids, Proceedings of SIGGRAPH 2001, 185-194.

2. Catmull E, Clark J, Recursively Generated B-spline Surfaces on Arbitrary Topological Meshes, Computer-Aided Design 10, 6, 350-355, 1978.

3. Cheng F, Yong J, Subdivision Depth Computation for Catmull-Clark Subdivision Surfaces, Computer Aided Design Es Applications 3, 1-4, 2006.

4. Cheng F, Chen G, Yong J, Subdivision Depth Computation for ExtraOrdinary Catmull-Clark Subdivision Surface Patches (complete version), www.cs.uky.edu/ cheng/PUBL/sub_depth_2.pdf

5. Halstead M, Kass M, DeRose T, Efficient, Fair Interpolation Using Catmull-Clark Surfaces, Proceedings of SIGGRAPH 1993, 35-44. 
6. Lai S, Cheng F, Parametrization of General Catmull-Clark Subdivision Surfaces and its Applications, Computer Aided Design 85 Applications 3, 1-4, 2006.

7. Litke N, Levin A, Schröder P, Trimming for Subdivision Surfaces, Computer Aided Geometric Design 18, 5, 463-481, 2001.

8. Peters J, Patching Catmull-Clark Meshes, Proceedings of SIGGRAPH 2000, 255-258.

9. Sederberg T W, Zheng J, Sewell D, Sabin M, Non-Uniform Recursive Subdivision Surfaces, Proceedings of SIGGRAPH 1998, 387-394.

10. Stam J, Exact Evaluation of Catmull-Clark Subdivision Surfaces at Arbitrary Parameter Values, Proceedings of SIGGRAPH 1998, 395-404.

11. Stam J, Evaluation of Loop Subdivision Surfaces, SIGGRAPH'99 Course Notes, 1999.

12. Wu X, Peters J, An Accurate Error Measure for Adaptive Subdivision Surfaces, Proc. Shape Modeling International 2005, 1-6.

13. Zorin, D., Schröder, P., and Sweldens, W. Interactive Multiresolution Mesh Editing. Proceedings of SIGGRAPH 1997, 259-268.

14. Zorin D, Kristjansson D, Evaluation of Piecewise Smooth Subdivision Surfaces, The Visual Computer, 18(5/6):299-315, 2002. 